\title{
Dilatación de la papila de Vater con balón de gran diámetro para la extracción de coledocolitiasis
}

\author{
J. Espinel y E. Pinedo ${ }^{1}$ \\ Servicios de Aparato Digestivo y ${ }^{\prime}$ Radiodiagnóstico. Hospital de León
}

\section{RESUMEN}

Objetivo: valorar la eficacia y seguridad de la dilatación de la papila de Vater con balones de gran diámetro (BGD) para la extracción de coledocolitiasis en pacientes con factores que dificultan o hacen peligrosa la extracción, por las características de los cálculos o de la anatomía peripapilar.

Diseño: prospectivo.

Pacientes: estudio que incluye 93 pacientes a los que se realizó dilatación hidrostática de la papila con balones de gran diámetro entre junio de 2005 y enero de 2008 por presentar cálculos de gran tamaño, múltiples, colédoco distal afilado, papila peri/intradiverticular, esfinterotomía previa o Billroth-II. Se emplearon dilatadores CRE de diámetros entre 12 y $20 \mathrm{~mm}$.

Resultados: se consiguió la extracción de los cálculos en una sesión en todos los pacientes (100\%). La mayor parte de las exploraciones (86\%) no requirieron tiempos prolongados para la extracción. Se precisó litotricia en el 3,2\%. Hubo dos complicaciones leves (2,1\%). Se detectó hiperamilasemia en el 16\% de los pacientes.

Conclusiones: la dilatación de la papila de Vater con balón de gran diámetro es una técnica eficaz y segura en la extracción de cálculos difíciles de la vía biliar, sin incrementar el tiempo de la exploración, ni las complicaciones, evitando la necesidad de litotricia en la mayoría de los pacientes.

Palabras clave: Dilatación de la papila de Vater. Coledocolitiasis. Colangiopancreatografía retrógrada endoscópica (CPRE). Complicaciones post-CPRE. Pancreatitis. Hiperamilasemia.

\begin{abstract}
Aim: to assess the efficacy and safety of dilatation of the papilla of Vater with large balloons for the treatment of choledocolithiasis in patients with difficult or risky extraction due to stone characteristics or peripapillary anatomy.

Design: prospective.

Patients: this study includes 93 patients in whom large-balloon dilation was performed between June 2005 and January 2008. Patients had multiple large stones, tapered distal CBD (common bile duct), peri-/intra-diverticular papilla, and previous sphincterotomy or Billroth-II surgery. A controlled radial expansion (CRE) balloon with a diameter range of $12-20 \mathrm{~mm}$ was used.

Results: stone removal was achieved in a single session in all patients (100\%). Most procedures (86\%) did not require an extended exploration time. Mechanical lithotripsy was needed in 3.2 $\%$ of cases. There were two mild complications (2.1\%). Hyperamilasemia was detected in $16 \%$ of patients.

Conclusions: papillary dilation with a large balloon is an effective, safe, and easy technique for the retrieval of difficult common bile-duct stones. The procedure neither adds time to the exploration, nor increases complications, and obviates the need for mechanical lithotripsy in a majority of patients.
\end{abstract}

Key words: Papillary dilation. Large ballon. Choledocolithiasis. Endoscopic retrograde cholangio-pancreatography (ERCP). PostERCP complications. Pancreatitis. Hyperamilasemia.

Espinel J, Pinedo E. Dilatación de la papila de Vater con balón de gran diámetro para la extracción de coledocolitiasis. Rev Esp Enferm Dig 2008; 100: 632-636.

Recibido: 29-04-08

Aceptado: 02-09-08.

Correspondencia: J. Espinel Díez. C/ Brianda de Olivera, 13, esc. 2, $3^{\circ}$ B. 24005 León. e-mail: jespinel@telefonica.net

\section{INTRODUCCIÓN}

La esfinterotomía endoscópica (EE) es el procedimiento de elección en la extracción de cálculos de la vía biliar. La dilatación de la papila de Vater (DP) fue descrita inicialmente en 1982 (1), aunque no se consideró una verdadera opción terapéutica hasta diez años después. La 
DP es una alternativa eficaz a la EE, consiguiéndose altos porcentajes de extracción de coledocolitiasis $(85-100 \%)$ y una menor incidencia de perforación y hemorragia (28). Si bien, algunas publicaciones han advertido un mayor riesgo de pancreatitis (4,9-12), este dato no se ha confirmado en otras $(8,13,14)$. Son aún escasos los estudios publicados respecto a la utilización de balones dilatadores de gran diámetro (DGD) en la extracción de coledocolitiasis (15-25). Estos dilatadores, permiten alcanzar calibres de 12 a $20 \mathrm{~mm}$ y, por lo tanto, pueden facilitar la extracción de cálculos difíciles. Nuestro estudio preliminar, puso de manifiesto su eficacia y seguridad (15). El objetivo del presente estudio ha sido valorar la eficacia de la dilatación de la papila de Vater con balones de gran diámetro (DBGD) en la extracción de coledocolitiasis en pacientes con factores que pudieran hacerla difícil o peligrosa, por las características de los cálculos (gran tamaño, múltiples) o de la anatomía peripapilar (colédoco distal afilado, papila peri/intradiverticular, esfinterotomía previa, Billroth-II). Se analizan los porcentajes de extracción, número de sesiones, empleo de litotricia, tiempo de exploración y las complicaciones aparecidas.

\section{PACIENTES Y MÉTODO}

\section{Pacientes}

Estudio prospectivo que incluye 93 pacientes (40 varones, 53 mujeres; edad media: 76,5 años; rango 43-94) entre junio de 2005 y enero de 2008. Cuarenta pacientes estaban colecistectomizados (43\%). Los criterios para realizar DBGD fue la extracción de coledocolitiasis con alguna de las siguientes características: cálculos de gran tamaño ( $\geq 15 \mathrm{~mm}$ ), múltiples ( $\geq 2$ ), colédoco distal afilado o cónico (desproporción entre el diámetro del cálculo y colédoco distal), esfinterotomía previa, papila peri/intradiverticular, Billroth-II. Se obtuvo el consentimiento informado de todos los pacientes.

\section{Método}

Todos los pacientes recibieron sedación profunda (propofol) controlada por un anestesista. Se administró profilaxis antibiótica, que se mantuvo durante las siguientes 24-48 h. La CPRE se realizó con un duodenoscopio terapéutico (TJF-140, Olympus). Tras la canulación de la vía biliar se realizó la colangiografía. En caso de imposibilidad en la canulación se realizó un precorte papilar. Una vez conseguido el acceso a la vía biliar se realizó una esfinterotomía convencional, empleando una corriente de 120 wat, endocut (ERBE). No se realizó esfinterotomia en los pacientes con Billroth-II, ni se amplió la esfinterotomía en los pacientes que tenían una esfinterotomía previa. Se emplearon dilatadores CRE de diámetros entre 12 y $20 \mathrm{~mm}$. El diámetro del balón se eligió de acuerdo con el tamaño del cálculo y del colédoco proximal al segmento afilado (sin sobrepasar el diámetro mayor del colédoco proximal). La dilatación de la papila fue progresiva, empleándose contraste diluido bajo control endoscópico y fluoroscópico, asegurando la correcta posición transpapilar y observando la gradual desaparición de la hendidura del esfínter de colédoco en el balón (Fig. 1). A continuación, el balón se mantuvo durante 30-45 segundos hasta ser retirado. Los cálculos se extrajeron con cesta de Dormia y/o balón de Fogarty, reservando el empleo de cesta de litotricia mecánica en los casos de imposibilidad de extracción con las anteriores técnicas. No se colocó prótesis pancreática como profilaxis de pancreatitis al finalizar el procedimiento en ningún paciente. Se realizó un hemograma y determinación de amilasa sérica a las 6 y $24 \mathrm{~h}$ del procedimiento y, se siguió la evolución de los pacientes hasta el alta, contactando telefónicamente a los 30 días para comprobar la aparición de complicaciones extrahospitalarias según criterios establecidos (26). Se consideró hiperamilasemia cuando existió aumento de al menos 3 veces los valores normales de la amilasa sérica en las $24 \mathrm{~h}$ posteriores. La duración de la CPRE se consideró: corta $(<15 \mathrm{~min})$; normal (15-30 min); prolongada (30-45 min); muy laboriosa (> $45 \mathrm{~min}$ ).

\section{RESULTADOS}

No se efectuó esfinterotomía en los pacientes que mostraban esfinterotomía previa (42 pacientes) o Billroth-II (4 pacientes). Se consiguió la extracción de los cálculos en una sola sesión en todos los pacientes (100\%). Tres pacientes precisaron litotricia mecánica $(3,2 \%)$. Los resultados y hallazgos de la CPRE se muestran en la tabla I. La duración de las exploraciones fue inferior a 45 minutos en 80 pacientes (86\%) (Fig. 2). El diámetro de los balones utilizados se muestra en la figura 3. Hubo dos complicaciones leves $(2,1 \%)$ : pancreatitis y hemorragia papilar, que se controló mediante tratamiento endoscópico con clip. Se detectó hiperamilasemia en 15 pacientes $(16 \%)$.

\section{DISCUSIÓN}

La dilatación del esfínter biliar y del colédoco distal con balon de gran diámetro, es un valioso complemento en el arsenal terapéutico que el endoscopista de la vía biliar puede utilizar en la extracción de coledocolitiasis en pacientes seleccionados. Cuando la realización de una adecuada esfinterotomia pueda ser difícil o peligrosa (papila peri/intradiverticular, esfinterotomia previa, BillrothII), o cuando el tamaño de la esfinterotomía es insuficiente para la extracción de coledocolitiasis de gran tamaño, la posterior ampliación del orificio de la esfinterotomía biliar con un balón de gran diámetro, puede aumentar el porcentaje de extracción, reducir o eliminar la necesidad 

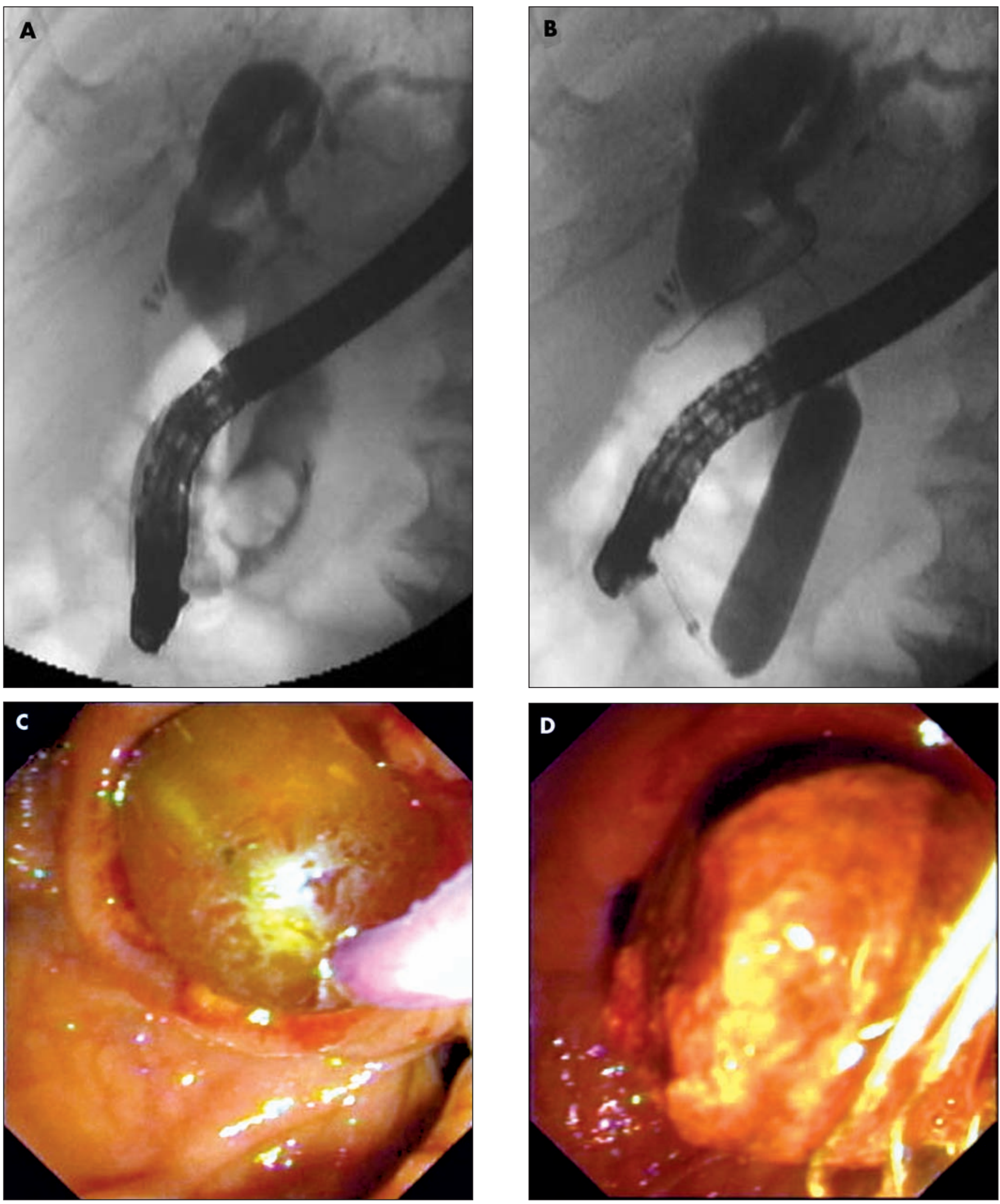

Fig. 1. A: Coledocolitiasis de gran tamaño (colangiografía); B: Balón dilatador de gran diámetro (imagen radiológica); C: Balón dilatador de gran diámetro (visión endoscópica); y D: Extracción del cálculo. 
Tabla I. Resultados y hallazgos de la DBGD

\begin{tabular}{|c|c|}
\hline & $n=93$ \\
\hline $\begin{array}{l}\text { Extracción de cálculos, n (\%) } \\
\text { Litotricia, } n(\%) \\
\text { Complicaciones, } n(\%) \\
\text { Hiperamilasemia, } n(\%)\end{array}$ & $\begin{array}{l}93(100) \\
3(3,2) \\
2(2,1) \\
15(16)\end{array}$ \\
\hline $\begin{array}{l}\text { Esfinterotomía previa, } \mathrm{n}(\%) \\
\text { Billroth-II, } \mathrm{n}(\%) \\
\text { Papila intra/peridiverticular, } \mathrm{n}(\%) \\
\varnothing \quad \text { Diámetro de colédoco proximal, media (rango) mm }\end{array}$ & $\begin{array}{c}42(45) \\
4(4,3) \\
30(32,2) \\
16,1(5-30)\end{array}$ \\
\hline $\begin{array}{l}\varnothing \quad \text { Diámetro de colédoco distal, media (rango) mm } \\
\text { Tamaño medio de los cálculos (rango) mm } \\
\text { Cálculos múltiples }(\geq 2), n(\%)\end{array}$ & $\begin{array}{c}8,5(4-16) \\
13,4(5-30) \\
60(64,5)\end{array}$ \\
\hline
\end{tabular}

DBGD: dilatación con balón de gran diámetro.

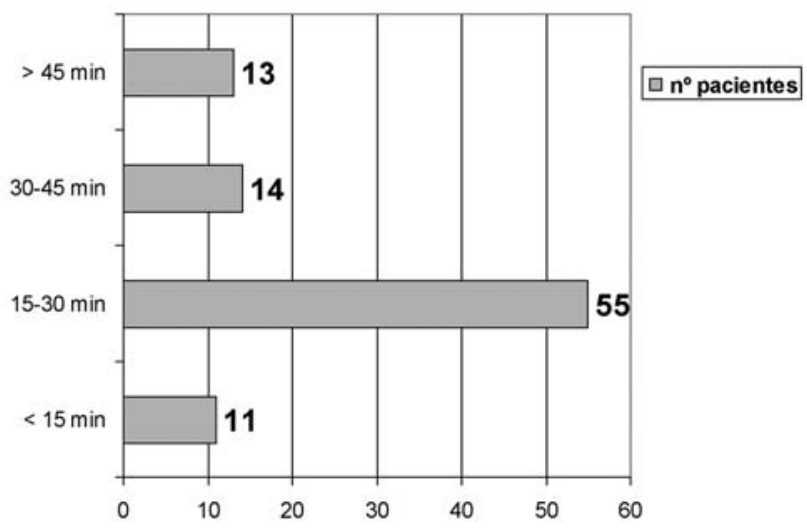

Fig. 2. Tiempos de las exploraciones.

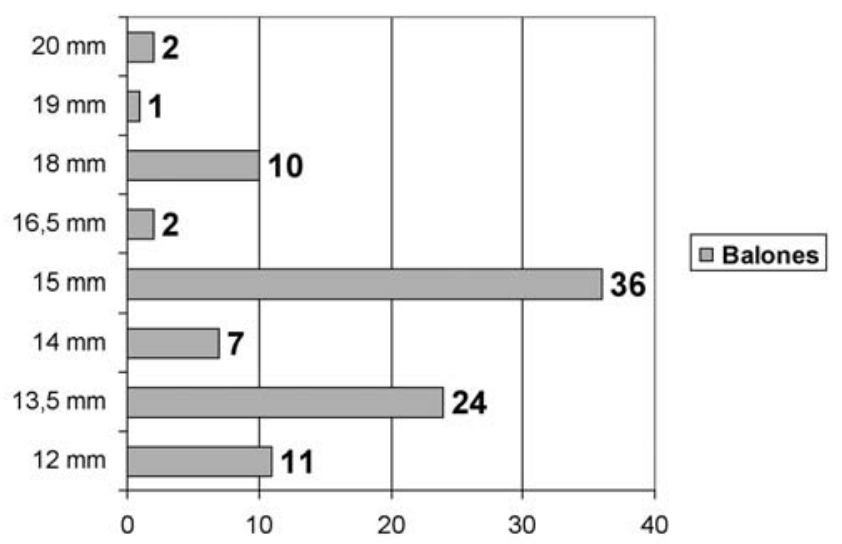

Fig. 3. Diámetros de los balones.

de realizar litotricia y, acortar el tiempo de la exploración (15-25). La realización de una esfinterotomía previa a la dilatación, permite separar el orificio pancreático del biliar. Es posible que la esfinterotomía preserve el orificio pancreático de la compresión del balón o libere fibras musculares entrelazadas con el esfínter pancreático, pudiendo reducir el riesgo de pancreatitis (24).

Existen pocos estudios clínicos publicados en los que se utilice esta técnica conjunta (esfinterotomia biliar más dilatación con balón de gran diámetro) en el tratamiento de coledocolitiasis de difícil extracción $(15,17-20,24)$. En estos estudios, el porcentaje de extracción de cálculos oscila entre el 83 y $100 \%$, y la tasa de complicaciones entre el 0 y el $16 \%$. En nuestro estudio preliminar, en el que se incluyeron 22 pacientes en los que el tamaño medio de los cálculos fue de $13 \pm 4 \mathrm{~mm}$, se consiguió la extracción completa en todos los casos, precisando solamente la realización de litotricia con cesta en un paciente (15). El presente estudio prospectivo pone de manifiesto que no es infrecuente advertir cálculos de difícil extracción por la desproporción entre el tamaño del cálculo y el diámetro del colédoco distal. Sin embargo, con esta técnica, los resultados son excelentes. El balón de gran diámetro tiene el efecto, al menos temporal, de ampliar el segmento distal del colédoco y abolir la función del esfínter, posibilitando la extracción de grandes cálculos, reduciendo la necesidad de realizar litotricia y acortando el tiempo de exploración. En nuestro estudio, solamente tres pacientes precisaron litotricia mecánica con cesta.

Respecto al tiempo de exploración, la mayor parte de las CPRE se realizaron en menos de 45 minutos. Unos tiempos que podemos considerar muy satisfactorios teniendo en cuenta que se trata de coledocolitiasis de difícil extracción. Este hecho, junto con la escasa necesidad de empleo de litotricia, puede reflejar una extracción menos traumática. El balón de gran diámetro consigue la dilatación del segmento distal de la vía biliar y del orificio papilar en una dirección, disminuyendo posiblemente el riesgo de perforación (18). No obstante, al igual que con otras técnicas emergentes donde la experiencia es limitada, serán necesarios todavía más estudios para establecer la seguridad de este procedimiento.

\section{CONCLUSIONES}

La dilatación de la papila de Vater con balón de gran diámetro (DBGD) es una técnica eficaz y segura en la extracción de cálculos difíciles de la vía biliar, sin incrementar el tiempo de la exploración, ni las complicaciones, evitando la necesidad de litotricia en la mayoría de los pacientes.

\section{BIBLIOGRAFÍA}

1. Staritz M, Ewe K, Meyer zum Büschenfelde KH. Endoscopic papillary dilatation, a possible alternative to endoscopic papillotomy. Lancet 1982; 1: 1306-7.

2. May GR, Cotton PB, Edmunds SEJ, Chong W. Removal of stones from the bile duct at ERCP winthout sphincterotomy. Gastrointest Endosc 1993; 39: 749-54.

3. Mac Mathuna P, White P, Clarke E, Lennon J. Crowe J. Endoscopic sphincteroplasty: a novel and safe alternative to papillotomy in the 
management of bile duct stones. Gut 1994; 35: 127-9.

4. Mac Mathuna P, White P, Clarke E, Merriman R, Lennon J, Crowe J. Endoscopic balloon sphincteroplasty (papillary dilation) for bile duct stones: efficacy, safety and follow-up in 100 patients. Gastrointest Endosc 1995; 42: 468-74.

5. Minami A, Nakatsu T, Uchida N, Hirabayashi S, Fukuma H, Morshed SA, et al. Papillary dilation vs sphincterotomy in endoscopic removal of bile duct stones. A randomized trial with manometric function. Dig Dis Sci 1995; 40: 2550-4

6. Komatsu Y, Kawabe T, Toda N, Ohashi M, Isayama M, Tateishi K, et al. Endoscopic papillary balloon dilation for the management of common bile duct stones: experience of 226 cases. Endoscopy 1998; 30: 12-7.

7. Espinel J, Muñoz F, Vivas S, Domínguez A, Linares P, Jorquera F, et al. Dilatación de la papila de Vater en el tratamiento de la coledocolitiasis en pacientes seleccionados. Gastroenterol Hepatol 2004; 27: 610.

8. Park do H, Kim MH, Lee SK, Lee SS, Choi JS, Song MH, et al. Endoscopic sphincterotomy vs. endoscopic papillary balloon dilation for choledocholitiasis in patients with liver cirrhosis and coagulopathy. Gastrointest Endosc 2004; 60: 180-5.

9. Kozarek RA. Balloon dilatation of the sphincter of Oddi. Endoscopy 1988; 20: 207-10

10. Disario JA, Freeman ML, Bjorkman DJ, Macmathuna P, Petersen BT, Jaffe PE, et al. Endoscopic balloon dilation compared with sphincterotomy for extraction of bile duct stones. Gastroenterology 2004; 127: 1291-9.

11. Baron TH, Harewood GC. Endoscopic balloon dilation of the biliary sphincter compared to endoscopic biliary sphincterotomy for removal of common bile duct stones during ERCP: a metaanalysis of randomized, controlled trials. Am J Gastroenterol 2004: 99: 1455-60.

12. Arnold JC, Benz C, Martin WR, Adamek HE, Riemann JF. Endoscopic papillary balloon dilation vs. sphincterotomy for removal of common bile duct stones: A prospective randomized pilot study. Endoscopy 2001; 33: 563-7.

13. Bergman JJ, Rauws EA, Fockens P, van Berkel AM, Bossuyt PM, Tijssen JG, et al. Randomised trial of endoscopic balloon dilation versus endoscopic sphincterotomy for removal of bile duct stones. Lancet 1997; 349: 1124-9.

14. Tanaka S, Sawayama T, Yoshioka T. Endoscopic papillary balloon dilation and endoscopic sphincterotomy for bile duct stones: longterm outcomes in a prospective randomized controlled trial. Gastrointest Endosc 2004; 59: 614-8.

15. Espinel J, Pinedo E, Olcoz JL. Large hydrostatic balloon for choledocolithiasis. Rev Esp Enferm Dig 2007; 99: 33-8.

16. Espinel J, Pinedo E, Calleja JL, Olcoz JL. Large balloon in the management of bile duct stones. Endoscopy 2007; 39 (Supl. I): A372.

17. Minami A, Hirose S, Nomoto T, Hayakawa S. Small sphincterotomy combined with papillary dilation with large balloon permits retrieval of large stones without mechanical lithotripsy. World J Gastroenterol 2007; 13: 2179-82.

18. Maydeo A, Bhandari S. Balloon sphincteroplasty for removing difficult bile duct stones. Endoscopy 2007; 11: 958-61.

19. Yoo B, Kim J, Jung J. Large-balloon sphincteroplasty along with or without sphincterotomy in patients with large extrahepatic bile duct stones: a multicenter study [abstract]. Gastrointest Endosc 2007; 65: AB97.

20. Attasaranya S, Cheon YK, McHenry L. Large-diameter papillary balloon dilation to aid in endoscopic bile duct stone removal: a multicenter series [abstract]. Gastrointest Endosc 2007; 65: AB214.

21. Misra SP, Dwivedi M. Large-diameter balloon dilation after endoscopic sphincterotomy for removal of difficult bile duct stones. Endoscopy 2008; 40: 209-13.

22. Cha SW, Hoon G, Choi G, Kim A, Yang H, Lee Y, et al. Endoscopic large balloon sphincterotomy for removal of large bile duct stone in patients with high risk of endoscopic sphincterotomy related complication. Endoscopy 2006; 38 (Supl. II): A232.

23. Heo JH, Kang DH, Jung HJ, Kwon DS, An JK, Kim BS, et al. Endoscopic sphincterotomy plus large-balloon dilation versus endoscopic sphincterotomy for removal of bile-duct stones. Gastrointest Endosc. 2007; 66 (4): 720-6.

24. Ersoz G, Tekesin O, Ozutemiz AO, Gunsar F. Biliary sphincterotomy plus dilation with a large balloon for bile duct stones that are difficult to extract. Gastrointest Endosc 2003; 57: 156-9.

25. Bang S. Endoscopic papillary balloon dilation with large balloon after partial endoscopic schincterotomy for retrieval of choledocholithiasis. Endoscopy 2005; 37 (Supl. I): A279.

26. Cotton PB, Lehman G, Vennes J, Geenen JE, Russell RCG, Meyers WC, et al. Endoscopic sphincterotomy complications and their management: an attempt at consensus. Gastrointest Endosc 1991; 37: 383-93. 